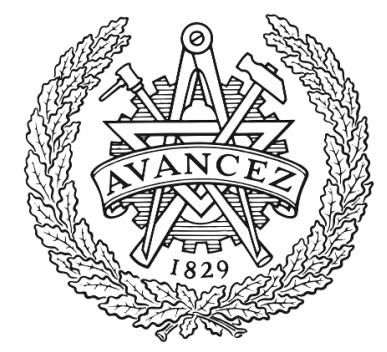

CHALMERS

UNIVERSITY OF TECHNOLOGY

\title{
Light-Off in Plasmon-Mediated Photocatalysis
}

Downloaded from: https://research.chalmers.se, 2023-04-26 10:38 UTC

Citation for the original published paper (version of record):

Tiburski, C., Boje, A., Nilsson, S. et al (2021). Light-Off in Plasmon-Mediated Photocatalysis. ACS Nano, 15(7): 11535-11542. http://dx.doi.org/10.1021/acsnano.1c01537

N.B. When citing this work, cite the original published paper. 


\title{
Light-Off in Plasmon-Mediated Photocatalysis
}

\author{
Christopher Tiburski, Astrid Boje, Sara Nilsson, Zafer Say, Joachim Fritzsche, Henrik Ström, \\ Anders Hellman, and Christoph Langhammer*
}

Cite This: ACS Nano 2021, 15, 11535-11542

Read Online

ABSTRACT: In plasmon-mediated photocatalysis it is of critical importance to differentiate light-induced catalytic reaction rate enhancement channels, which include near-field effects, direct hot carrier injection, and photothermal catalyst heating. In particular, the discrimination of photothermal and hot electron channels is experimentally challenging, and their role is under keen debate. Here we demonstrate using the example of $\mathrm{CO}$ oxidation over nanofabricated neat $\mathrm{Pd}$ and $\mathrm{Au}_{50} \mathrm{Pd}_{50}$ alloy catalysts, how photothermal rate enhancement differs by up to 3 orders of magnitude for the same photon flux, and how this effect is controlled

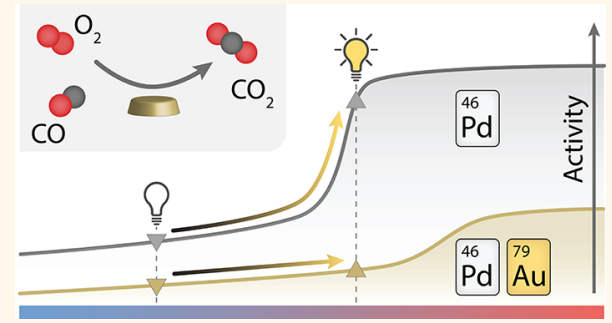
solely by the position of catalyst operation along the light-off curve measured in the dark. This highlights that small fluctuations in reactor temperature or temperature gradients across a sample may dramatically impact global and local photothermal rate enhancement, respectively, and thus control both the balance between different rate enhancement mechanisms and the way strategies to efficiently distinguish between them should be devised.

KEYWORDS: nanoalloys, heterogeneous catalysis, plasmonics, photothermal, CO oxidation, gold-palladium, photocatalysis

$\mathrm{P}$ lasmon-mediated photocatalysis has seen rapid development since the seminal publications more than a decade ago. ${ }^{1-9}$ It builds on the excitation of the localized surface plasmon resonance (LSPR) in metal nanoparticles and the fact that, in this way, optical cross sections many times larger than the particle size can be achieved. ${ }^{10}$ Mechanistically, the LSPR phenomenon may influence a chemical process on a metal nanoparticle surface in three distinctly different ways, which, in principle, may manifest themselves individually or in concert: (i) photothermal heat generation, (ii) optical nearfield enhancement, and (iii) direct hot-charge carrier generation in the metal and their injection into surfaceadsorbed reactant species. ${ }^{11-13}$ Ever since the hot-carrier mechanism was first proposed, one of the most important and critical questions has been how to distinguish it from the photothermal one. As a consequence, being able to appropriately answer this question has developed into a key effort in the field, spurred significantly by a recent controversy on data interpretation in key studies, ${ }^{13-18}$ and discussions of experimental procedures for distinguishing photothermal from hot-carrier reaction enhancement processes. 19,20

Focusing on the photothermal mechanism, we remind ourselves that upon decay of an LSPR in a metal nanoparticle, the excited electrons will relax via electron-phonon coupling within picoseconds, unless steered otherwise. ${ }^{21,22}$ In other words, the electronic excitation is transferred into heat via coupling to the phonon bath of the nanoparticle, which further dissipates the heat to the nanoparticle surrounding and support, and reaches thermal equilibrium on the $10-100 \mathrm{ps}$ time scale. ${ }^{18,21}$ As a consequence, temperature gradients may form due to (i) nonuniform metal nanoparticle distributions, (ii) inhomogeneous illumination intensity, (iii) 3D nano- or mesoporous support materials with complex and anisotropic heat transport properties, and (iv) attenuation/absorption of irradiated photons inside such a support material. These effects have been identified as important and pinpointed as inadequately addressed in the literature. ${ }^{14,18}$

A further important factor in this context is the fact that the reaction rate of a thermally activated catalytic reaction does not depend linearly on temperature. In the kinetic regime, when the rate is relatively low, its temperature dependence can be described by the Arrhenius equation, which states that the rate constant is defined by $k=A \exp \left(-E_{\mathrm{a}} / \mathrm{RT}\right)$, where $A$ is the preexponential factor, $E_{\mathrm{a}}$ the molar activation energy, $R$ the gas constant, and $T$ temperature. As a first key aspect, this means that the reaction rate increases exponentially with temperature. Consequently, in a photothermal process, provided the reaction is taking place in the kinetically limited regime, the rate is expected to exhibit an exponential dependence on the illumination power. $^{20}$ Therefore, the most widespread approach to distinguish between photothermal and direct hot-carrier mediated reaction mechanisms has been to study a reaction at increasing photon flux and investigate whether this

Received: February 19, 2021

Accepted: June 15, 2021

Published: June 22, 2021

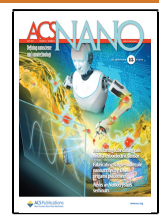


dependence is linear or exponential-an approach that has been identified as problematic and to yield ambiguous results. $^{20}$ The main reason for this ambiguity is that in corresponding studies only limited ranges of photon flux variation have been used since actually implementing the required several orders of magnitude difference in photon flux would require the use of very high-power light sources and/or the use of ultrasensitive detection techniques at very low reaction rates.

As a second key aspect, we note that in a real system the increase of the reaction rate cannot continue to infinity, since, at a certain point, diffusion limitations and mass transport gradients will start to dominate the process. This leads to a slowing down, and eventually a complete halt, of the rate increase, once the system is operated entirely in the mass transport limited regime. In thermal catalysis, this behavior is captured in a so-called light-off curve, which typically is a plot of the reaction rate (or conversion) as a function of temperature. As the key implication here, the photothermal enhancement will be strongly dependent on where on the lightoff curve the catalyst finds itself during the experiment. In other words, the same photon flux can lead to rate enhancements that differ by several orders of magnitude, depending on the starting temperature of the catalyst. Consequently, for example, small fluctuations in reactor temperature, as well as temperature gradients across a sample, may have a dramatic impact on the (local) photothermal rate enhancement and the balance between the three potential plasmonic rate enhancement mechanisms when they take place in concert. Surprisingly, however, this aspect is rarely addressed in the debate on enhancement mechanisms in plasmonmediated catalysis and the corresponding discussion of experimental strategies to efficiently distinguish between them.

In response, we report here a study that explicitly demonstrates the importance and potential magnitude of this effect on the example of carbon monoxide (CO) oxidation over two-dimensional (2D) nanofabricated $\mathrm{Pd}$ and $\mathrm{Pd}_{50} \mathrm{Au}_{50}$ alloy model catalysts. We have chosen these specific systems because they by design effectively eliminate $3 \mathrm{D}$ temperature gradients within the catalyst material due to their $2 \mathrm{D}$ nature and because alloying $\mathrm{Pd}$ with $\mathrm{Au}$ constitutes an efficient way to alter the light-off behavior of the reaction, ${ }^{23,24}$ while, as we demonstrate, essentially not altering light absorption, and thus the photothermal properties of the system.

\section{RESULTS AND DISCUSSION}

Sample Characterization. For our study, we have nanofabricated large-area arrays of $\mathrm{Pd}$ and $\mathrm{Pd}_{50} \mathrm{Au}_{50}$ alloy nanoparticles by hole-mask colloidal lithography ${ }^{25,26}$ onto 18 $\mathrm{mm} \times 9 \mathrm{~mm}$ fused silica substrates (Figure $1 \mathrm{a}$ and $\mathrm{b}$ ). The resulting $\mathrm{Pd}$ and $\mathrm{Pd}_{50} \mathrm{Au}_{50}$ nanoparticles have a disk shape with an average diameter of 113 and $132 \mathrm{~nm}$ and a height of 38 and $28 \mathrm{~nm}$, respectively (see Methods for details). Transmission electron microscopy-energy dispersive X-ray analysis (TEMEDX) reveals homogeneous and uniform alloy formation across the $\mathrm{Pd}_{50} \mathrm{Au}_{50}$ particles (Figure $1 \mathrm{c}$ ). ${ }^{26} \mathrm{X}$-ray photoelectron spectroscopy (XPS) furthermore reveals a surface composition of 46 at \% Au and 54 at \% Pd, which is in good agreement with the targeted value, and due to the surface sensitivity of XPS, again also confirms homogeneous alloy formation (Figure 1d).

CO Oxidation in the Dark. For the catalysis experiments, we have utilized an externally heated quartz-tube plug-flow- a
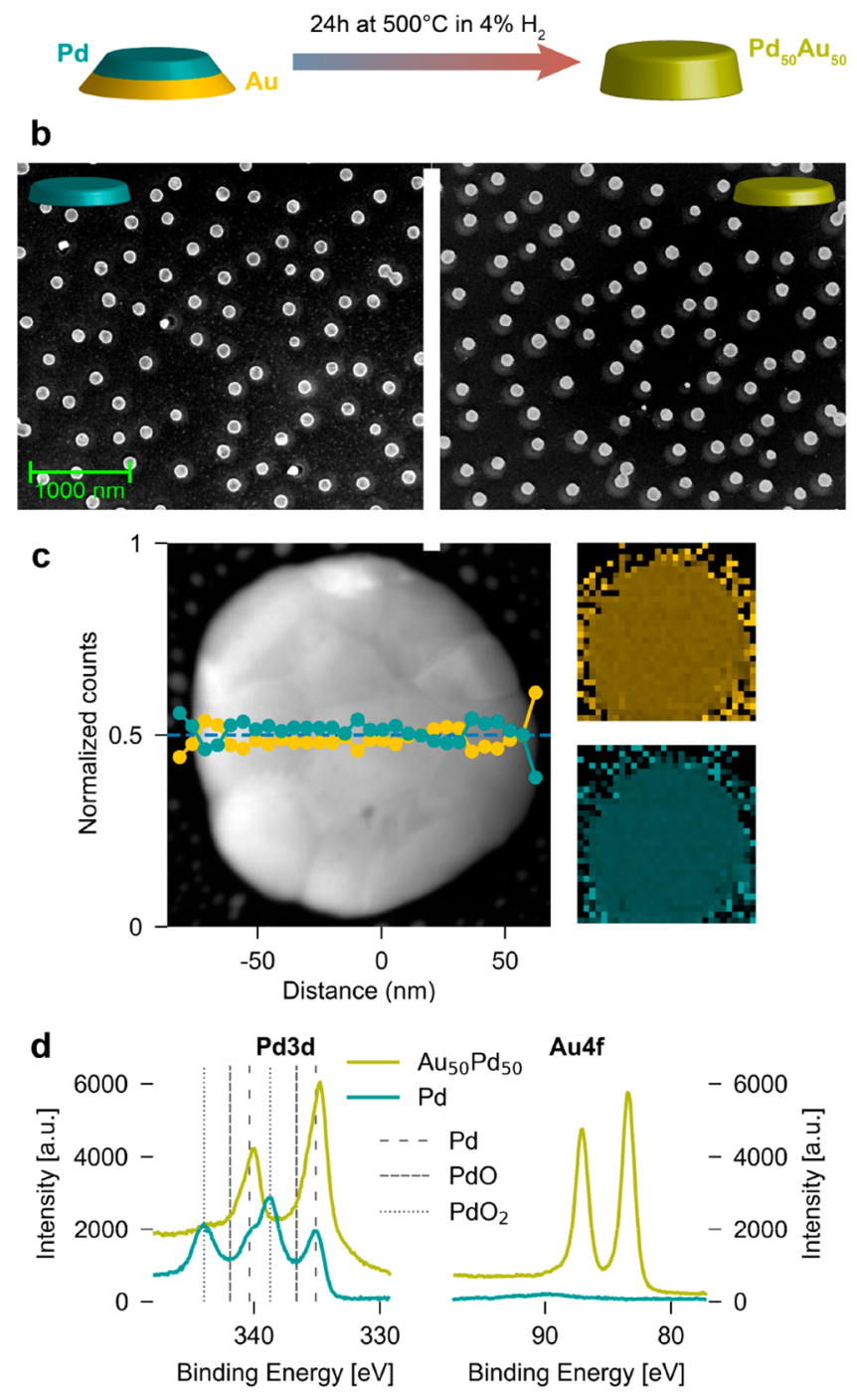

Figure 1. $\mathrm{Pd}$ and $\mathrm{Au}_{50} \mathrm{Pd}_{50}$ sample characterization. (a) The alloy nanoparticle array is manufactured by subsequently evaporating a thin $\mathrm{Au}$ and $\mathrm{Pd}$ film through a nanofabricated mask, with thicknesses tailored to yield the targeted alloy composition. Subsequently, alloy formation is induced by annealing the sample at $500{ }^{\circ} \mathrm{C}$ for $24 \mathrm{~h}$ in $4 \% \mathrm{H}_{2}$ in Ar carrier gas. ${ }^{26}$ (b) SEM images of annealed neat $\mathrm{Pd}(\mathrm{left})$ and $\mathrm{Au}_{50} \mathrm{Pd}_{50}$ alloy (right) nanodisk arrays. (c) HAADF-STEM image of an $\mathrm{Au}_{50} \mathbf{P d}_{50}$ alloy nanodisk, depicted together with both an elemental line scan and a TEM-EDS map of the $\mathrm{Pd}$ and $\mathrm{Au}$ constituents. The line scan confirms the homogeneous distribution of both elements across the particle. (d) XPS spectra of a neat $\mathbf{P d}$ and an $\mathrm{Au}_{50} \mathbf{P d}_{50}$ alloy nanoparticle array taken before catalysis experiments for the Pd $3 \mathrm{~d}$ (left) and Au $4 f$ (right) peak regions, revealing a surface composition of 46 at $\% \mathrm{Au}$ and 54 at \% Pd for the alloy, as well as some degree of PdO and $\mathrm{PdO}_{2}$ formation (Figures $\mathrm{S} 1$ and $\mathrm{S} 2$ ) corroborated also by SEM imaging (Figure S3).

type reactor with an integrated "glass pocket", as reported by Fredriksson et al. $^{27,28}$ This pocket minimizes dilution of reaction products and thereby enables quadrupole mass spectrometric (QMS) analysis of reaction products from our nanofabricated 2D samples (Figure S4). The CO oxidation experiments were conducted with a total gas flow rate of 200 $\mathrm{mL} / \mathrm{min}$, which introduces a flow rate of $2.4 \mathrm{~mL} / \mathrm{min}$ through the pocket, in a gas mixture of $\mathrm{O}_{2}$ and $\mathrm{CO}$ in $\mathrm{Ar}$ carrier at a 
constant relative $\mathrm{CO}$ concentration $\alpha^{\mathrm{CO}}=\frac{C_{\mathrm{CO}}}{C_{\mathrm{CO}}+C_{\mathrm{O} 2}}=0.2$ and a total reactant concentration of $10 \%$. During the experiment, the sample temperature in the dark (i.e., without illumination) was increased in $\sim 10{ }^{\circ} \mathrm{C}$ steps from $\sim 150$ to $\sim 325^{\circ} \mathrm{C}$ (Figure S5). Temperature control was implemented by setting a constant power output of the heating system, that is, without using an active feedback loop. This is important since an active feedback system would reduce the heating power supplied during illumination to compensate for the light-induced heating of the sample. To ensure a constant temperature at each temperature step, we instead equilibrated the system for 150 min (Figure S5). By then extracting the CO conversion at the end of each temperature step, we constructed the light-off curves for neat $\mathrm{Pd}$ and the $\mathrm{Au}_{50} \mathrm{Pd}_{50}$ alloy system in the dark (Figure 2). In the low temperature regime, where the reaction

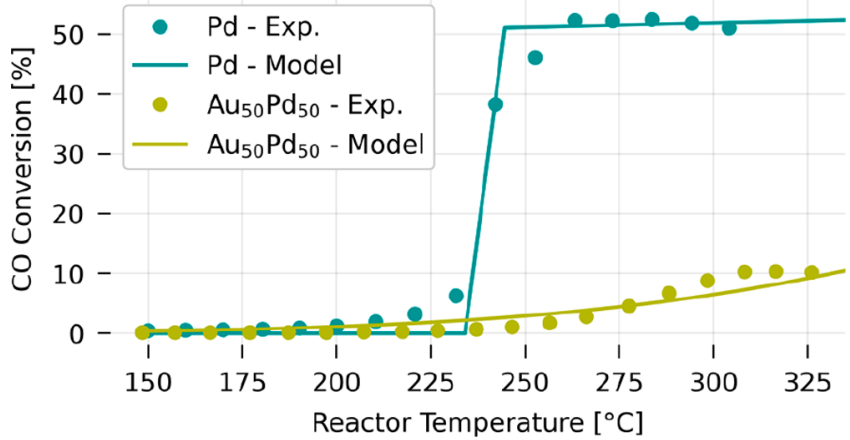

Figure 2. $\mathrm{Pd}$ and $\mathrm{Au}_{50} \mathrm{Pd}_{50}$ light-off curves in the dark. The light-off curves for an array of neat $\mathbf{P d}$ and $\mathrm{Au}_{50} \mathbf{P d}_{50}$ alloy nanoparticles were obtained using a gas flow rate of $200 \mathrm{~mL} / \mathrm{min}$ in a gas mixture of $\mathrm{O}_{2}$ and $\mathrm{CO}$ in $\mathrm{Ar}$ carrier at a constant relative $\mathrm{CO}$ concentration $\alpha_{\mathrm{CO}}=0.2$ and a total reactant concentration of $10 \%$, by increasing sample temperature in the dark in $\sim 10^{\circ} \mathrm{C}$ steps from $\sim 150$ to $\sim 325{ }^{\circ} \mathrm{C}$ (see also Figure S5). The solid lines show the conversion predictions for a CSTR model (see the Kinetics section of the SI) using the same conditions as in the experiment (Table S4), i.e., a residence time of $4.5 \mathrm{~s}$ and inlet pressure of $1 \mathrm{~atm}$. The Pd catalyst model was a $\mathbf{P d}(111)$ surface and the $\mathrm{Au}_{50} \mathbf{P d}_{50}$ model was a mixed PdAu surface with an Au top layer (see Tables S1 and S2). The number of active sites in each model system was chosen to match the maximum experimentally observed conversion since the exact number of sites in the experiment is unknown.

is slow enough not to be limited by mass transport, the reaction rate is kinetically limited. Upon further temperature increase, the system reaches and passes through a transient regime, where a transition from kinetically controlled to mass transport-controlled conditions takes place. This yields the typical S-like light-off curve for both systems. However, as the key result we highlight that the transition to mass-transport control occurs at lower temperature for the neat Pd catalyst compared to the $\mathrm{Pd}_{50} \mathrm{Au}_{50}$ system and that conversion for the latter is generally significantly reduced.

In a first analysis step to rationalize the differing light-off behavior observed between $\mathrm{Pd}$ and $\mathrm{Au}_{50} \mathrm{Pd}_{50}$ in Figure 2, we conducted first-principles informed microkinetic modeling. Specifically, since the exact surface structure of the alloy used in the experiment is unknown and the surface may undergo rearrangement in the presence of adsorbates, we studied three simplified configurations with DFT (details in the firstprinciples and kinetics calculations section of the SI and structures in Tables S1 and S2) and found a configuration with an $\mathrm{Au}$ top layer to be most thermodynamically favorable, followed by a perfectly mixed $\mathrm{Pd}-\mathrm{Au}$ surface. However, in reality a blend of configurations is likely to coexist on the alloy catalyst, with local reactivity depending on the presence of $\mathrm{Au}$ and $\mathrm{Pd}$ atoms at the surface. Next, we calculated the adsorption energies of $\mathrm{CO}$ and oxygen on the three chosen surface configurations and found reduced binding energies for the systems with $\mathrm{Au}$ atoms in the top layer (Table S3). Then, we predicted the transition state energies for $\mathrm{CO}$ oxidation using the scaling relation derived by Falsig et al., ${ }^{29}$ indicating a reduced activation energy with increasing presence of $\mathrm{Au}$ atoms on the surface (Table S3).These weaker binding energies and reduced barriers with increasing Au fraction are consistent with previous first-principles calculations of the PdAu alloy system. ${ }^{30}$

First-Principles Informed Microkinetic Modeling. To now investigate the impact of these different energetics on the expected light-off behavior of the catalyst, we modeled the pocket reactor used in the experiments as a continuously stirred tank reactor (CSTR) with residence time and conditions chosen to match the experimental setup (details in the Kinetics section of the SI and Table S4). Specifically, we tested the energetics obtained for each modeled surface configuration and estimated the unknown exact number of active sites in the experiment to match the experimentally observed maximal conversion for each system. The correspondingly obtained results for the neat Pd surface reproduce the measured conversion profile very well (Figure 2). For the alloy system, we obtained the best agreement with the experiment for the most thermodynamically favorable model surface (i.e., the surface with an Au top layer), which showed reduced surface concentrations of reactants and correspondingly lower conversion. Smoother conversion profiles, lacking a sharp light-off point, were obtained for the model systems with $\mathrm{Au}$ atoms in the top surface layer due to the reduced kinetic barriers in these systems. This suggests that both reduced binding energies and reaction barriers for the alloy system, consistent with gold atoms near the surface, explain the differences in the magnitude and shape of the conversion profiles observed for the neat $\mathrm{Pd}$ and $\mathrm{Au}_{50} \mathrm{Pd}_{50}$ systems. Taken together, the first-principles calculations and kinetic modeling results thus provide a qualitative rationalization for the observed conversion profiles based on the respective energetics. However, we also highlight that they do not explicitly describe the likely dynamic surface restructuring taking place in the real catalyst (to do so a more complex model would be required, which is beyond the scope of this work) and thus do not mean that a continuous Au top layer is formed. Rather, they should be interpreted as that an Au-rich surface is formed and the reason for the experimentally observed differences between the pure $\mathrm{Pd}$ and the $\mathrm{Au}_{50} \mathrm{Pd}_{50}$ light-off curves.

CO Oxidation under Illumination. Having established the $\mathrm{CO}$ oxidation reaction on our two catalyst model systems in the dark, we now assess their interaction with visible light. Specifically, we carried out finite-difference time-domain (FDTD) simulations of the absorption efficiency of $\mathrm{a}_{x} \mathrm{Pd}_{1-x}$ alloy nanodisk with its composition, $x$, varied in steps of 10 at $\%$ from neat $\mathrm{Pd}$ to $\mathrm{Au}_{50} \mathrm{Pd}_{50}$, using dielectric functions calculated from first-principles as the input (Figure 3a). ${ }^{31}$ In good agreement with our previous results assessing extinction efficiencies, ${ }^{31}$ increasing the Au content in the alloy shifts the LSPR absorption peak to lower photon energies. Furthermore, 


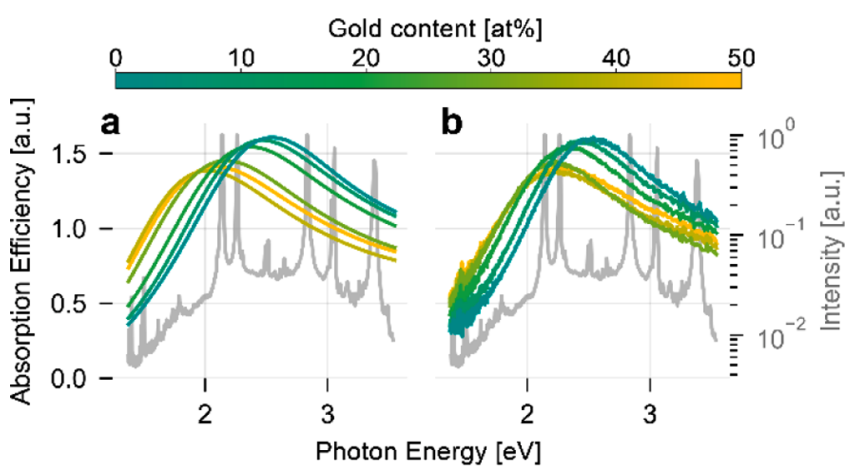

C

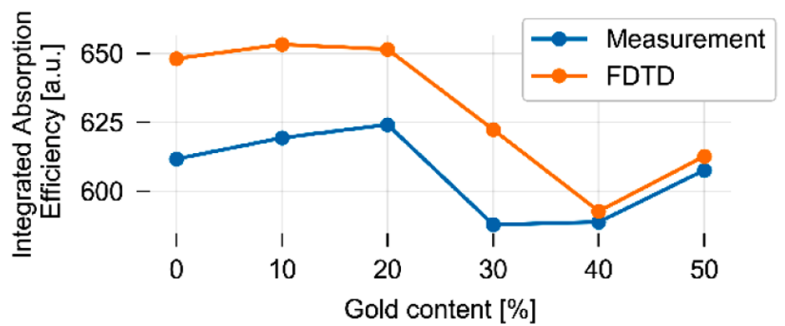

Figure 3. Light absorption efficiency in Pd and AuPd alloy nanodisks. (a) Absorption efficiency (absorption cross-section/ nanodisk projected area) spectra for a nanodisk comprised of neat $\mathrm{Pd}$ and $\mathrm{Au}_{x} \mathrm{Pd}_{100-x}$ alloy with its composition varied in $x=10$ at $\%$ steps up to a composition of $\mathrm{Au}_{50} \mathbf{P d}_{50}$. The spectra were obtained by FDTD simulations for a single nanodisk located on a fused silica substrate and by using the dielectric functions from the work of Rahm et al. as input. ${ }^{31}$ The gray line plotted in the background depicts the emission spectrum of the light source used for the photocatalysis experiments described below and confirms excellent overlap with the absorption spectra. (b) Corresponding experimentally measured absorption efficiency spectra for quasirandom arrays of neat $\mathrm{Pd}$ and $\mathrm{PdAu}$ alloy nanodisks with composition varied in 10 at \% steps up to a composition of $\mathrm{Au}_{50} \mathrm{Pd}_{50}$. The experimental data are scaled to the FDTD data (for unscaled spectra, see Figure S6). (c) Integrated absorption efficiencies calculated from the spectra depicted in $a$ and $b$ for the spectral range from 1.37 to $3.55 \mathrm{eV}$. The total absorbed energy varies only by around $12 \%$, which very likely results in a similar photon-induced temperature increase of the nanostructures upon illumination, irrespective of their composition.

and as the key point here, the overall appearance of the peak, i.e., the full-width-at-half-maximum that is proportional to the LSPR dephasing time, ${ }^{21,22}$ remains very similar throughout the entire composition range. Nanofabricating arrays of $\mathrm{Pd}$ and $\mathrm{Au}_{x} \mathrm{Pd}_{1-x}$ alloy nanoparticles across the same compositional range, and experimentally measuring their absorption efficiencies (defined as absorption cross section/nanodisk projected area derived from SEM image analysis) using an integrating sphere detector, ${ }^{10,32}$ reveals good agreement with the simulations (Figure $3 \mathrm{~b}$ ) and further corroborates the similar total absorption efficiencies of neat $\mathrm{Pd}$ and its alloys with $\mathrm{Au}$ up to 50 at \%. This last point becomes even more clear when plotting the integrated absorption efficiencies for Pd and its alloys as obtained both from the FDTD simulations and the experimental data (Figure 3c). Specifically, this analysis shows that the overall light absorption of an array of $\mathrm{Pd}$ and $\mathrm{Au}_{50} \mathrm{Pd}_{50}$ alloy nanoparticles is similar and, therefore, is expected to yield a similar photothermal heating effect due to light absorption, while at the same time having significantly different catalytic properties in the dark.
Turning now to assessing the impact of visible light illumination on our two model catalyst systems during reaction, we carried out an experiment where we measured the reaction rate of the two systems in the dark and under illumination of a mercury xenon arc light source at constant output irradiance of $6.8 \mathrm{~W} / \mathrm{cm}^{2}$ for five selected temperatures $\left(150,170,190,210,220{ }^{\circ} \mathrm{C}\right)$ along the light-off curve (cf. Figure 2). As the main result, we find a clear rate enhancement under illumination for both catalyst systems, which strongly depends on the catalyst temperature in the dark, with the dependence being distinctly nonlinear and significantly lower for the $\mathrm{Au}_{50} \mathrm{Pd}_{50}$ alloy (Figure 4a). Further quantification of
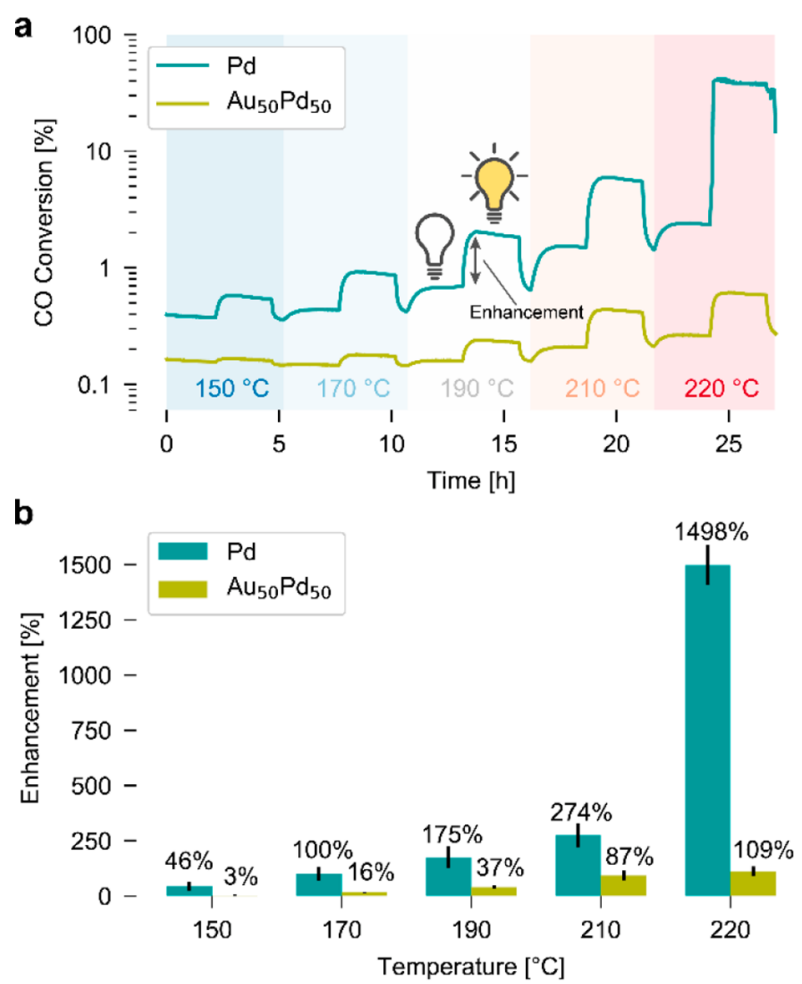

Figure 4. Light-induced reaction rate enhancement for constant irradiance at different catalyst temperatures. (a) The $\mathrm{CO}$ conversion in the dark and upon illumination for neat Pd (blue) and $\mathrm{Au}_{50} \mathbf{P d}_{50}$ (yellow) measured in a constant flow of $\mathrm{CO}$ and $\mathrm{O}_{2}$ in Ar carrier gas with a relative $\mathrm{CO}$ concentration, $\alpha{ }^{\mathrm{CO}}=\frac{C_{\mathrm{CO}}}{\mathrm{C}_{\mathrm{CO}}+\mathrm{C}_{\mathrm{O} 2}}$, of 0.2 and at five different dark temperatures ranging from $\sim 150$ to $\sim 220{ }^{\circ} \mathrm{C}$. (b) The enhancement of the reaction rate due to constant-irradiance illumination of the two model catalysts at five different temperatures in the dark. The black lines constitute error bars that depict the standard deviation from three repetitions. Note the strong dependence of the rate enhancement on catalyst dark temperature, and the significant difference of this effect between neat $\mathbf{P d}$ and the $\mathrm{Au}_{50} \mathbf{P d}_{50}$ alloy system.

the enhancement effect, defined as $\frac{I_{\mathrm{CO}_{2}, \text { llluminated }}-I_{\mathrm{CO}_{2} \text {,dark }}}{I_{\mathrm{CO}_{2} \text {,dark }}} \times 100$, reveals that for neat $\mathrm{Pd}$ at $220^{\circ} \mathrm{C}$ the reaction rate is enhanced by $\sim 1500 \%$ upon illumination, whereas the enhancement is only $\sim 50 \%$ at $150{ }^{\circ} \mathrm{C}$ (Figure $4 \mathrm{~b}$ ). For the $\mathrm{Au}_{50} \mathrm{Pd}_{50}$ system the overall trend is similar, however, at $150{ }^{\circ} \mathrm{C}$ the light-induced rate enhancement is only $3 \%$ and at $220^{\circ} \mathrm{C}$ it is $110 \%$, which is more than a factor 10 lower compared to neat $\mathrm{Pd}$.

It is now interesting to discuss these findings from a number of different perspectives. First of all, from a purely plasmonics 
point of view, this result may appear surprising because it is generally well-established that Au is a "better" plasmonic metal than Pd. ${ }^{22,33}$ Hence, one could intuitively expect that adding $\mathrm{Au}$ to $\mathrm{Pd}$ would boost the plasmon-mediated catalytic properties. ${ }^{34}$ Interestingly, however, we observe the opposite. Second, as we have shown (cf. Figure 3), the integrated absorption efficiency of the $\mathrm{Pd}$ and $\mathrm{Au}_{50} \mathrm{Pd}_{50}$ systems is very similar, which implies that any (photothermal) enhancement effects would be similar as well. Clearly, also this scenario is in stark contrast to our experimental results.

To nevertheless understand our findings, we first remind ourselves of the light-off curves measured for both model catalysts in the dark. They revealed a significantly lower thermal rate for the $\mathrm{Au}_{50} \mathrm{Pd}_{50}$ alloy compared to neat $\mathrm{Pd}$ for constant temperature (cf. Figure 2). Second, we extract the $\mathrm{CO}$ conversion for each of the five reactor temperatures in the dark and under illumination (Figure S7), together with the corresponding illumination-induced sample temperature increase measured with a thermocouple that touches the side of the sample (Figure S4b), which are on the order of $25{ }^{\circ} \mathrm{C}$ (Figure S8). Plotting the correspondingly obtained $\mathrm{CO}$ conversion for each temperature step under illumination

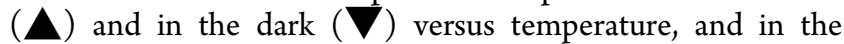
same graph as the light-off curve previously measured in the dark (cf. Figure 2), we make the following key observations (Figure 5): (i) light-induced rate enhancement is strongly

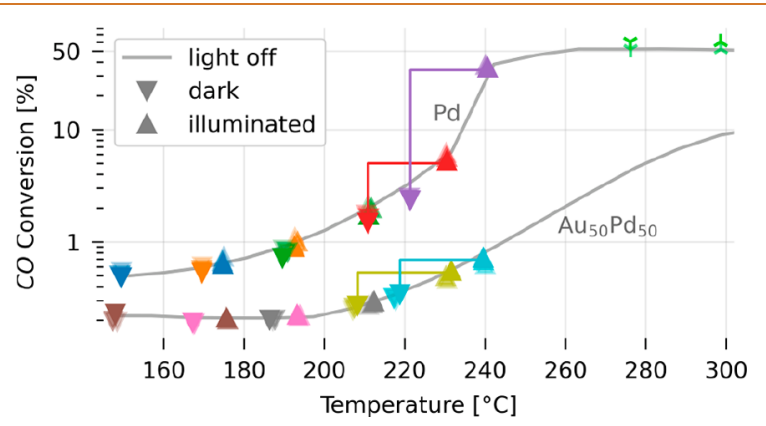

Figure 5. CO conversion in the dark and under illumination. CO conversion for five temperature steps under illumination $(\boldsymbol{\Delta})$ and in the dark $(\nabla)$ plotted versus temperature and in the same graph as the light-off curve previously measured in the dark (calibration-cf. Figure 2). The semitransparent markers represent repeated measurements. The colored lines indicate the shift of the catalyst along the light-off curve upon illumination. Also shown are two data points obtained in the full mass transport limited regime in a separate measurement under illumination $(\lambda)$ and in the dark $(Y)$, which reveal that in that limit no light-induced conversion enhancement is observed, as expected (see Figure S9 for raw data).

dependent on the reactor temperature; (ii) the light-induced enhancement is significantly smaller for the $\mathrm{Au}_{50} \mathrm{Pd}_{50}$ catalyst compared to neat $\mathrm{Pd}$, irrespective of reactor temperature; (iii) the $\mathrm{CO}$ conversion values measured upon illumination align almost perfectly with $\mathrm{CO}$ conversion values of the light-off curve measured in the dark for both catalysts, irrespective of the reactor temperature; (iv) at high temperature, in the fully mass-transport limited regime, we do not observe any lightinduced rate enhancement. Altogether, this clearly shows that it is the position along the light-off curve, determined by the set reactor temperature, which dictates the observed photothermal reaction rate enhancement. Furthermore, it demonstrates that the apparent significantly lower photoactivity of the
$\mathrm{Au}_{50} \mathrm{Pd}_{50}$ alloy is solely the consequence of its intrinsically lower catalytic activity in the dark at the present conditions. These observations are further corroborated by the fact that we actually observe a slightly reduced light-induced sample temperature increase for higher reactor temperatures from the thermocouple reading for both samples (from $\sim 26$ to $\sim 20$ ${ }^{\circ} \mathrm{C}$-Figure S8), while we see that the light-induced reaction rate enhancement is increased. As a final remark, we also note that the thermal equilibration between the catalyst nanoparticles and the support is very efficient even for the used fused silica support and that, therefore, thermal gradients between the particles and the support can be neglected. ${ }^{35}$

\section{CONCLUSIONS}

In conclusion, we have shown that the photothermal reaction rate enhancement on nanofabricated $\mathrm{Pd}$ and $\mathrm{Au}_{50} \mathrm{Pd}_{50}$ alloy model catalysts during the catalytic oxidation of $\mathrm{CO}$ strongly depends on the catalyst temperature in the dark, and is solely mediated by its light-off curve. Specifically, we have found that the photothermal rate enhancement for the Pd system varies over 3 orders of magnitude at constant irradiance, depending on the position of the catalyst on the light-off curve. Simultaneously, we have identified that photothermal rate enhancements for the $\mathrm{Au}_{50} \mathrm{Pd}_{50}$ alloy system are significantly lower, despite very similar integrated optical absorption efficiency and thus light-induced temperature increase. As the reason we identified the overall lower catalytic activity of the alloy system compared to neat $\mathrm{Pd}$, and the consequently significantly different light-off curve of the reaction in the dark. To explain this different catalytic behavior, we conducted firstprinciples informed microkinetic modeling showing that the lower activity of $\mathrm{Au}_{50} \mathrm{Pd}_{50}$ is caused by reduced binding energies of $\mathrm{CO}$ and oxygen, which leads to low surface coverages of both reactants, hindering the kinetic activity despite a corresponding reduction in the activation energy.

As the key implications of the above results, we highlight that understanding the light-off curve of a catalyst is critical when assessing any plasmon-mediated photocatalysis effects. Thus, as explicitly demonstrated here, a catalyst's sensitivity toward a photothermal rate enhancement mechanism may vary by multiple orders of magnitude at constant irradiance, depending solely on the systems position along the light-off curve. Consequently, when more realistic three-dimensional catalyst systems are considered, locally, the catalyst may be operated at different positions along a light-off curve due to (i) temperature gradients across the material, (ii) attenuation of the photon flux within the sample, or (iii) locally varying mass transport limitations within a porous support. This, in turn, means that locally the balance between reaction enhancement mechanisms may be very different, further complicating discrimination between them. Finally, our results also highlight the importance of light-off characterization in plasmonmediated catalysis when designing experimental strategies for discriminating rate enhancement mechanisms since the photothermal channel can be dramatically suppressed or enhanced, depending on the position along the light-off curve. Therefore, it should be carefully selected and optimized for each specific catalyst and reaction system.

\section{METHODS}

Nanofabrication. All samples were fabricated using hole-mask colloidal lithography ${ }^{36,37}$ on either fused silica for catalysis experiments and spectrophotometry, on oxidized silicon for SEM imaging, 
or on TEM windows ${ }^{38}$ for TEM-EDS measurements. For the nanofabrication steps the following instrumentation was used: spincoating (Suss, LabSpin6), oxygen ion etching (Plasmatherm, BatchTop m/95), electron beam evaporation (Lesker, PVD 225). To create the hole-mask, we used a 0.2 wt \% a $140 \mathrm{~nm}$ sulfate latex bead solution (molecular probes by life technologies) in deionized water. The nanodisks were annealed in a flow reactor for $24 \mathrm{~h}$ at 500 ${ }^{\circ} \mathrm{C}$ in $(4 \pm 0.12) \% \mathrm{H}_{2}$ in $\mathrm{Ar}$ carrier gas at a flow rate of $200 \mathrm{~mL} / \mathrm{min}$ to induce alloy formation. The annealing process leads further to a slight change in dimensions (Figure S10).

Plug-Flow Reactor with "Pocket". The (photo)catalysis experiments were carried out in a plug-flow type reactor (X1, Insplorion $A B$, Göteborg, Sweden) equipped with a custom-made pocket reactor as reported by Fredriksson et al. (Figure S4). ${ }^{27,28}$ Gas composition in and flow rate through the reactor is controlled by mass flow controllers (Bronkhorst Low- $\Delta \mathrm{P}$ ). The reactor is heated by a resistive heating coil and connected to a quadrupole mass spectrometer (Pfeiffer, GSD 320). Two type-K thermocouples are inserted in the reactor to monitor (i) the temperature of the gas upstream of the pocket and (ii) the actual temperature of the sample. The latter thermocouple is spring-loaded to ensure good contact with the side of the sample inside the pocket. The thermocouple is placed in the light path and therefore experiences a constant temperature increase upon illumination, which, however, is on the order of $1{ }^{\circ} \mathrm{C}$ or less (Figure S11) and the same for all experiments and catalysts. Furthermore, this heating of the thermocouple does not impact the temperature control of the reactor since the temperature reading is not used in a feed-back loop manner. The reactor has an optical port perpendicular to the flow direction to enable illumination by a $1000 \mathrm{~W}$ mercury xenon arc light source (Newport 6295NS in a 66921 housing), which is equipped with a liquid optical IR filter (Newport 6123NS).

Before the photocatalysis experiments, the samples were activated by a treatment of $60 \mathrm{~min} 6 \% \mathrm{O}_{2}$, followed by $30 \mathrm{~min} 3 \% \mathrm{H}_{2}$, and finally $60 \mathrm{~min} 6 \% \mathrm{O}_{2}$ in Ar carrier gas with a constant flow of $200 \mathrm{~mL} /$ min at $500{ }^{\circ} \mathrm{C}$. The activation was followed by a cooling period of $5 \mathrm{~h}$ to $150{ }^{\circ} \mathrm{C}$ in Ar. Then, $1.5 \mathrm{~h}$ prior to the first temperature step or temperature ramp, $\mathrm{CO}$ and $\mathrm{O}_{2}$ were introduced simultaneously.

The used gases were $\mathrm{CO}(10 \%( \pm 2 \mathrm{rel} \%)$ in $\mathrm{Ar}), \mathrm{O}_{2}(99.9992 \%$ purity), $\mathrm{H}_{2}$ (4\% ( \pm 2 rel \%) in $\left.\mathrm{Ar}\right)$, and $\mathrm{Ar}$ ( $99.9999 \%$ purity) as the carrier gas.

FDTD Simulations. Lumericals FDTD solution version 8.21.2088 was utilized to calculate the optical absorption spectra presented in Figure 3. A single disk was placed on a $\mathrm{SiO}_{2}$ support with a refractive index of $1.46 .{ }^{39}$ The dielectric function for $\mathrm{Pd}$ and the $\mathrm{Au}_{x} \mathrm{Pd}_{100-x}$ alloys with $x=10,12,30,40,50$ were taken from Rahm et al. ${ }^{31}$ The disk diameter (Figure S10) used in the simulations was derived from SEM images by analyzing at least 350 particles from different sample positions and averaging their individual diameters. The particle itself was modeled as a tapered cylinder (angle of $5^{\circ}$ ) with rounded edges ( $4 \mathrm{~nm}$ bottom and $6 \mathrm{~nm}$ top rounding). The used disk height was calculated by assuming the conservation of volume from the unannealed to the annealed particle (Figure S10). The linearly polarized plane wave light source was introduced via a total-field/ scattered field source.

X-ray Photoelectron Spectroscopy. XPS measurements were performed with a PHI 5000 (Physical Electronics). The photoexcitation was done with a monochromatized $\mathrm{K} \alpha$-line of an $\mathrm{Al}$ source operated at $50 \mathrm{~W}$. The energy step width was $0.1 \mathrm{eV}$, and the pass energy was $55 \mathrm{eV}$. The base pressure was always lower than $2.0 \times$ $10^{-6} \mathrm{~Pa}$. All spectra were corrected by setting the adventitious C-1s peak of the $\mathrm{C}-\mathrm{C}$ bond to $284.8 \mathrm{eV}$. To ensure that this correction is physically meaningful, the Fermi level alignment was checked as suggested by Greczynski and Hultman. ${ }^{40}$ For quantitative analysis relative sensitivity factors from PHI MultiPak's database for Au and Pd of 417.021 and 341.244, respectively, were employed.

Spectrophotometry. The optical absorption spectra were obtained with a Cary 5000 spectrophotometer (Varian) equipped with a DRA2500 integrating sphere accessory. The spectra were recorded from 250 to $2500 \mathrm{~nm}$ with a step width of $1 \mathrm{~nm}$.
Scanning Electron Microscopy. A Zeiss Supra 55 was used to record the SEM images with the in-lens system and an acceleration voltage of $15 \mathrm{kV}$ at a working distance of at least $5 \mathrm{~mm}$.

Transmission Electron Microscopy. For the STEM images and the elemental EDS maps a FEI Titan 80-300 equipped with an INCA $\mathrm{X}$-sight detector (Oxford Instruments) was used. It was operated at $300 \mathrm{kV}$ and lateral resolution was $5 \mathrm{~nm}$ for which the spectra were acquired. The acquisition time was $5 \mathrm{~s}$ for each spectrum, and the sample holder was tilted about $20^{\circ}$ toward the detector to increase Xray signal. The background was corrected for and the peaks fitted standard-less using the FEI TIA software version 4.3.

First-Principles Calculations. Periodic density functional theory calculations were used to find relaxed geometries and energies for $\operatorname{Pd}(111)$ and three PdAu alloy surfaces. Calculations were performed on $2 \times 2 \times 3$ unit cells using VASP, ${ }^{41,42}$ with the revised $\mathrm{PBE}^{43}$ generalized gradient approximation and projector augmented wave method. ${ }^{44}$ An energy cutoff of $450 \mathrm{eV}$ was used for the plane wave basis set. The three PdAu surfaces comprised a perfect mix of Pd and $\mathrm{Au}$ atoms on the lower layers with (i) a perfectly mixed top layer, (ii) an $\mathrm{Au}$ top layer and Pd second layer, and (iii) a Pd top layer and $\mathrm{Au}$ second layer. Binding energies were calculated for $\mathrm{CO}$ and $\mathrm{O}$ on each surface and the gas molecules were relaxed in a $10 \times 10 \times 10 \AA^{3}$ box. More information is given in the first-principles and kinetics calculations section of the SI, with structures in Tables S1 and S2, and energies in Figure S13 and Table S3.

Microkinetic Model. Modeled surface kinetics included CO adsorption, $\mathrm{O}_{2}$ dissociative adsorption, and irreversible $\mathrm{CO}$ oxidation to form $\mathrm{CO}_{2} \cdot \mathrm{CO}_{2}$ desorption was assumed to be instantaneous and irreversible. $\mathrm{CO}$ and $\mathrm{O}_{2}$ desorption were assumed to be in thermodynamic equilibrium with the respective adsorption steps. The binding energies from the DFT calculations were used as the reaction energies for adsorption and to predict the activation energies for $\mathrm{CO}$ oxidation on each surface using the scaling relation developed by Falsig and co-workers ${ }^{29}$ (Table S3). CO oxidation was modeled as a Langmuir-Hinshelwood reaction, with a prefactor from transition state theory. The free energies were computed in the harmonic limit for adsorbates and the ideal gas approximation for gases. The pocket reactor was modeled as a continuously stirred tank reactor with surface-based kinetics. The system of differential equations describing the surface kinetics and flow was solved in Python using the SciPy LSODA integrator ${ }^{45}$ and the BDF method, with relative and absolute tolerances of $1 \times 10^{-8}$ and $1 \times 10^{-10}$, respectively. More details and a discussion of model choices are provided in the Kinetics section of the SI, Table S4, and Figures S14 and S15.

\section{ASSOCIATED CONTENT}

\section{s! Supporting Information}

The Supporting Information is available free of charge at https://pubs.acs.org/doi/10.1021/acsnano.1c01537.

XPS spectrum of $\mathrm{Pd} 3 \mathrm{~d}$ of $\mathrm{Pd}$ and $\mathrm{Au}_{50} \mathrm{Pd}_{50}$, XPS spectrum of $\mathrm{Pd} 3 \mathrm{~d}$ and $\mathrm{Au} 4 \mathrm{f}$ prior $\mathrm{CO}$ oxidation, SEM images of the samples, schematic of the experimental setup, raw data of light-off curves, absorption efficiency spectra and integrated absorption efficiency for different alloy compositions, $\mathrm{CO}_{2}$ ion current in the dark and under illumination, temperature difference in the dark and under illumination of the sample at different temperature steps, $\mathrm{CO}_{2}$ ion current in the dark and under illumination in the mass transport limited regime, mean diameter and height of samples after annealing, temperature increase upon illumination, transmission spectrum of empty glass pocket and intensity profile of lamp, explanation of first-principle calculations, side view of relaxed $\mathrm{CO}$ and $\mathrm{O}$ adsorbates for different surface configurations, top view of relaxed $\mathrm{CO}$ and $\mathrm{O}$ adsorbates for different surface configurations, binding energies of $\mathrm{CO}$ and $\mathrm{O}$ calculated by $\mathrm{DFT}$, electronic and free 
energies calculated by DFT, explanation of considered $\mathrm{CO}$ oxidation kinetics, predicted conversion for different model systems, steady-state surface coverage as a function of temperature for different model systems (PDF)

\section{AUTHOR INFORMATION}

\section{Corresponding Author}

Christoph Langhammer - Department of Physics, Chalmers University of Technology, 41296 Göteborg, Sweden; (1) orcid.org/0000-0003-2180-1379; Email: clangham@ chalmers.se

\section{Authors}

Christopher Tiburski - Department of Physics, Chalmers University of Technology, 41296 Göteborg, Sweden

Astrid Boje - Department of Physics, Chalmers University of Technology, 41296 Göteborg, Sweden; (1) orcid.org/00000002-2487-0276

Sara Nilsson - Department of Physics, Chalmers University of Technology, 41296 Göteborg, Sweden

Zafer Say - Department of Physics, Chalmers University of Technology, 41296 Göteborg, Sweden

Joachim Fritzsche - Department of Physics, Chalmers University of Technology, 41296 Göteborg, Sweden

Henrik Ström - Department of Mechanics and Maritime Sciences, Chalmers University of Technology, 41296 Göteborg, Sweden; 이이. orcid.org/0000-0002-8581-5174

Anders Hellman - Department of Physics, Chalmers University of Technology, 41296 Göteborg, Sweden; (1) orcid.org/0000-0002-1821-159X

Complete contact information is available at: https://pubs.acs.org/10.1021/acsnano.1c01537

\section{Notes}

The authors declare no competing financial interest.

\section{ACKNOWLEDGMENTS}

This work was funded by the Knut and Alice Wallenberg Foundation projects 2016.0210 and 2015.0055. Part of this work was carried out at the MC2 cleanroom facility and at the Chalmers Materials Analysis Laboratory (CMAL).

\section{REFERENCES}

(1) Christopher, P.; Xin, H.; Linic, S. Visible-Light-Enhanced Catalytic Oxidation Reactions on Plasmonic Silver Nanostructures. Nat. Chem. 2011, 3, 467-472.

(2) Linic, S.; Aslam, U.; Boerigter, C.; Morabito, M. Photochemical Transformations on Plasmonic Metal Nanoparticles. Nat. Mater. 2015, 14, 567-576.

(3) Mukherjee, S.; Libisch, F.; Large, N.; Neumann, O.; Brown, L. V.; Cheng, J.; Lassiter, J. B.; Carter, E. A.; Nordlander, P.; Halas, N. J. Hot Electrons Do the Impossible: Plasmon-Induced Dissociation of $\mathrm{H}_{2}$ on Au. Nano Lett. 2013, 13, 240-247.

(4) Hou, W.; Cronin, S. B. A Review of Surface Plasmon ResonanceEnhanced Photocatalysis. Adv. Funct. Mater. 2013, 23, 1612-1619.

(5) Kale, M. J.; Avanesian, T.; Christopher, P. Direct Photocatalysis by Plasmonic Nanostructures. ACS Catal. 2014, 4, 116-128.

(6) Smith, J. G.; Faucheaux, J. A.; Jain, P. K. Plasmon Resonances for Solar Energy Harvesting: A Mechanistic Outlook. Nano Today 2015, $10,67-80$.

(7) Tian, Y.; Tatsuma, T. Plasmon-Induced Photoelectrochemistry at Metal Nanoparticles Supported on Nanoporous $\mathrm{TiO}_{2}$. Chem. Commun. 2004, 1810-1811.
(8) Nitzan, A.; Brus, L. E. Theoretical-Model for Enhanced Photochemistry on Rough Surfaces. J. Chem. Phys. 1981, 75, 22052214.

(9) Brus, L. Noble Metal Nanocrystals: Plasmon Electron Transfer Photochemistry and Single-Molecule Raman Spectroscopy. Acc. Chem. Res. 2008, 41, 1742-1749.

(10) Langhammer, C.; Kasemo, B.; Zoric, I. Absorption and Scattering of Light by Pt, Pd, Ag, and Au Nanodisks: Absolute Cross Sections and Branching Ratios. J. Chem. Phys. 2007, 126, 194702.

(11) Baffou, G.; Quidant, R. Nanoplasmonics for Chemistry. Chem. Soc. Rev. 2014, 43, 3898-3907.

(12) Seemala, B.; Therrien, A. J.; Lou, M.; Li, K.; Finzel, J. P.; Qi, J.; Nordlander, P.; Christopher, P. Plasmon-Mediated Catalytic $\mathrm{O}_{2}$ Dissociation on $\mathrm{Ag}$ Nanostructures: Hot Electrons or near Fields? ACS Energy Letters 2019, 4, 1803-1809.

(13) Dubi, Y.; Sivan, Y. Hot" Electrons in Metallic NanostructuresNon-Thermal Carriers or Heating? Light: Sci. Appl. 2019, 8, 89.

(14) Dubi, Y.; Un, I. W.; Sivan, Y. Thermal Effects - An Alternative Mechanism for Plasmon-Assisted Photocatalysis. Chemical Science 2020, 11, 5017-5027.

(15) Sivan, Y.; Baraban, J.; Un, I. W.; Dubi, Y. Comment on "Quantifying Hot Carrier and Thermal Contributions in Plasmonic Photocatalysis". Science 2019, 364, eaaw9367.

(16) Jain, P. K. Comment on "Thermal Effects - An Alternative Mechanism for Plasmon-Assisted Photocatalysis" by Y. Dubi, I. W. Un and Y. Sivan, Chemical Science, 2020, 11, 5017. Chemical Science 2020, 11, 9022-9023.

(17) Dubi, Y.; Un, I. W.; Sivan, Y. Reply to the 'Comment on "Thermal Effects-An Alternative Mechanism for Plasmon-Assisted Photocatalysis"' by P. Jain, Chemical Science, 2020, 11, DOI: 10.1039/D0SC02914A. Chem. Sci. 2020, 11, 9024-9025.

(18) Jain, P. K. Taking the Heat Off of Plasmonic Chemistry. J. Phys. Chem. C 2019, 123, 24347-24351.

(19) Sivan, Y.; Baraban, J. H.; Dubi, Y. Experimental Practices Required to Isolate Thermal Effects in Plasmonic Photo-Catalysis: Lessons from Recent Experiments. OSA Continuum 2020, 3, 483497.

(20) Baffou, G.; Bordacchini, I.; Baldi, A.; Quidant, R. Simple Experimental Procedures to Distinguish Photothermal from HotCarrier Processes in Plasmonics. Light: Sci. Appl. 2020, 9, 108.

(21) Bauer, M.; Marienfeld, A.; Aeschlimann, M. Hot Electron Lifetimes in Metals Probed by Time-Resolved Two-Photon Photoemission. Prog. Surf. Sci. 2015, 90, 319-376.

(22) Zoric, I.; Zäch, M.; Kasemo, B.; Langhammer, C. Gold, Platinum, and Aluminum Nanodisk Plasmons: Material Independence, Subradiance, and Damping Mechanisms. ACS Nano 2011, 5, $2535-2546$

(23) Venezia, A. M.; Liotta, L. F.; Pantaleo, G.; La Parola, V.; Deganello, G.; Beck, A.; Koppany, Z.; Frey, K.; Horvath, D.; Guczi, L. Activity of $\mathrm{SiO}_{2}$ Supported Gold-Palladium Catalysts in $\mathrm{CO}$ Oxidation. Appl. Catal., A 2003, 251, 359-368.

(24) Gao, F.; Goodman, D. W. Pd-Au Bimetallic Catalysts: Understanding Alloy Effects from Planar Models and (Supported) Nanoparticles. Chem. Soc. Rev. 2012, 41, 8009-8020.

(25) Fredriksson, H.; Alaverdyan, Y.; Dmitriev, A.; Langhammer, C.; Sutherland, D. S.; Zaech, M.; Kasemo, B. Hole-Mask Colloidal Lithography. Adv. Mater. 2007, 19, 4297-4302.

(26) Nugroho, F. A. A.; Iandolo, B.; Wagner, J. B.; Langhammer, C. Bottom-Up Nanofabrication of Supported Noble Metal Alloy Nanoparticle Arrays for Plasmonics. ACS Nano 2016, 10, 2871-2879.

(27) Fredriksson, H. O. A.; Larsson Langhammer, E. M.; Niemantsverdriet, J. W. Reduction of Cu-Promoted Fe Model Catalysts Studied by in Situ Indirect Nanoplasmonic Sensing and XRay Photoelectron Spectroscopy. J. Phys. Chem. C 2015, 119, 40854094.

(28) Bu, Y.; Niemantsverdriet, J. W. H.; Fredriksson, H. O. A. Cu Model Catalyst Dynamics and CO Oxidation Kinetics Studied by Simultaneous in Situ UV-Vis and Mass Spectroscopy. ACS Catal. 2016, 6, 2867-2876. 
(29) Falsig, H.; Hvolbaek, B.; Kristensen, I. S.; Jiang, T.; Bligaard, T.; Christensen, C. H.; Norskov, J. K. Trends in the Catalytic CO Oxidation Activity of Nanoparticles. Angew. Chem., Int. Ed. 2008, 47, $4835-4839$.

(30) Zhang, J.; Jin, H.; Sullivan, M. B.; Lim, F. C.; Wu, P. Study of Pd-Au Bimetallic Catalysts for CO Oxidation Reaction by DFT Calculations. Phys. Chem. Chem. Phys. 2009, 11, 1441-1446.

(31) Rahm, J. M.; Tiburski, C.; Rossi, T. P.; Nugroho, F. A. A.; Nilsson, S.; Langhammer, C.; Erhart, P. A Library of Late Transition Metal Alloy Dielectric Functions for Nanophotonic Applications. Adv. Funct. Mater. 2020, 30, 2002122.

(32) Langhammer, C.; Schwind, M.; Kasemo, B.; Zorić, I. Localized Surface Plasmon Resonances in Aluminum Nanodisks. Nano Lett. 2008, 8, 1461-1471.

(33) Langhammer, C.; Yuan, Z.; Zoric, I.; Kasemo, B. Plasmonic Properties of Supported Pt and Pd Nanostructures. Nano Lett. 2006, 6, 833-838.

(34) Sytwu, K.; Vadai, M.; Dionne, J. A. Bimetallic Nanostructures: Combining Plasmonic and Catalytic Metals for Photocatalysis. Advances in Physics: $X$ 2019, 4, 1619480.

(35) Zhdanov, V. P.; Zorić, I.; Kasemo, B. Plasmonics: Heat Transfer between Metal Nanoparticles and Supporting Nanolayers. Phys. E 2012, 46, 113-118.

(36) Fredriksson, H.; Alaverdyan, Y.; Dmitriev, A.; Langhammer, C.; Sutherland, D. S.; Zaech, M.; Kasemo, B. Hole-Mask Colloidal Lithography. Adv. Mater. 2007, 19, 4297-4302.

(37) Nugroho, F. A. A.; Iandolo, B.; Wagner, J. B.; Langhammer, C. Bottom-Up Nanofabrication of Supported Noble Metal Alloy Nanoparticle Arrays for Plasmonics. ACS Nano 2016, 10, 2871-2879.

(38) Grant, A. W.; Hu, Q. H.; Kasemo, B. Transmission Electron Microscopy 'Windows' for Nanofabricated Structures. Nanotechnology 2004, 15, 1175-1181.

(39) Palik, E. D. Handbook of Optical Constants of Solids; Academic press: Orlando, 1998; Vol. 3.

(40) Greczynski, G.; Hultman, L. X-Ray Photoelectron Spectroscopy: Towards Reliable Binding Energy Referencing. Prog. Mater. Sci. 2020, 107, 100591.

(41) Kresse, G.; Hafner, J. ab initio Molecular Dynamics for OpenShell Transition Metals. Phys. Rev. B: Condens. Matter Mater. Phys. 1993, 48, 13115-13118.

(42) Kresse, G.; Furthmuller, J. Efficiency of ab-Initio Total Energy Calculations for Metals and Semiconductors Using a Plane-Wave Basis Set. Comput. Mater. Sci. 1996, 6, 15-50.

(43) Hammer, B.; Hansen, L. B.; Norskov, J. K. Improved Adsorption Energetics within Density-Functional Theory Using Revised Perdew-Burke-Ernzerhof Functionals. Phys. Rev. B: Condens. Matter Mater. Phys. 1999, 59, 7413-7421.

(44) Blochl, P. E. Projector Augmented-Wave Method. Phys. Rev. B: Condens. Matter Mater. Phys. 1994, 50, 17953-17979.

(45) Petzold, L. Automatic Selection of Methods for Solving Stiff and Nonstiff Systems of Ordinary Differential-Equations. Siam Journal on Scientific and Statistical Computing 1983, 4, 136-148. 